\title{
Phase of the Fermion Determinant at Nonzero Chemical Potential
}

\author{
K. Splittorff ${ }^{1}$ and J.J.M. Verbaarschot ${ }^{1,2}$ \\ ${ }^{1}$ The Niels Bohr Institute, Blegdamsvej 17, DK-2100, Copenhagen Ø, Denmark \\ ${ }^{2}$ Department of Physics and Astronomy, SUNY, Stony Brook, New York 11794, USA
}

(Dated: September 24, 2018)

\begin{abstract}
We show that in the microscopic domain of QCD (also known as the $\epsilon$-domain) at nonzero chemical potential the average phase factor of the fermion determinant is nonzero for $\mu<m_{\pi} / 2$ and is exponentially suppressed for larger values of the chemical potential. This follows from the chiral Lagrangian that describes the low-energy limit of the expectation value of the phase factor. Explicit expressions for the average phase factor are derived using a random matrix formulation of the zero momentum limit of this chiral Lagrangian.
\end{abstract}

Introduction. During the past decade, a great deal of progress has been made in understanding the phase diagram of the QCD partition function in the chemical potential - temperature plane. Although early analytical arguments 1] clarified the nature of the chiral phase transition along the temperature axis, a detailed quantitative understanding could only be achieved by means of lattice QCD simulations (see 2] for a review). The situation at nonzero chemical potential is much less clear. Although perturbative arguments, model calculations and phenomenological arguments seem to give a consistent picture [3], first principle quantitative information is lacking. The main reason is that $\mathrm{QCD}$ at nonzero chemical potential cannot be simulated reliably in much of the chemical potential - temperature plane because the fermion determinant is complex (the sign problem). Progress has been made around the critical temperature and small chemical potentials, where different lattice QCD approaches seem to converge [4, 5, 6, 7, 8]. Throughout this letter, chemical potential is short for quark chemical potential and will be denoted by $\mu$.

The question we wish to address in this letter is whether there exists a parameter domain for which the phase of the fermion determinant is manageable. Since there is no sign problem for $\mu=0$, we expect that for sufficiently small nonzero chemical potential lattice QCD simulations are possible. The standard argument is that the number of gauge field configurations required for a converged calculation diverges exponentially with the volume as $\exp (V \Delta F)$, where $V \Delta F$ is the difference of the free energy of typical gauge field configurations generated by the Monte Carlo algorithm and the converged free energy. One limit in which the sign problem remains manageable is when $V \Delta F$ remains finite in the thermodynamic limit. We expect that for sufficiently small $\mu$ the free energy difference behaves as $\mu^{2} V$. For the sign problem to be manageable the chemical potential then has to scale as $1 / \sqrt{V}$ in the thermodynamic limit. Such type of limit is well known - it is the microscopic limit or the $\epsilon$-limit of the QCD partition function. In this limit the mass and chemical potential dependence of the QCD partition function is determined by the zero momentum modes of the Goldstones associated with the spontaneous breaking of chiral symmetry.

One observable that directly tests the severity of the sign problem is the expectation value of the phase factor of the fermion determinant. This phase factor can be expressed as the ratio of the fermion determinant and its complex conjugate

$$
e^{2 i \theta}=\frac{\operatorname{det}\left(D+\mu \gamma_{0}+m\right)}{\operatorname{det}\left(D^{\dagger}+\mu \gamma_{0}+m\right)}
$$

Its expectation value is a QCD-like partition function with a low energy limit that is determined along wellestablished rules by chiral symmetry and gauge invariance 9,10 . In this letter we analyze the average phase factor in the microscopic domain of QCD [11, 12, 13, 14]

$$
m_{\pi}^{2} \ll \frac{1}{\sqrt{V}}, \quad \mu^{2} \ll \frac{1}{\sqrt{V}} .
$$

In this domain the Compton wave length of the Goldstone modes is much larger than the linear size of the box, so that the chiral Lagrangian can be truncated to its zero momentum sector. In the thermodynamic limit, simple expressions can then be obtained using mean field arguments. At finite volume, the calculations are much more complicated, but we can exploit the equivalence with random matrix theory [12, 13], where recent progress 15, 16, 17, 18, 19, 20] makes it possible to derive exact results in the microscopic domain. Several cases will be discussed: the quenched limit, the phase quenched limit, and QCD with dynamical flavors. In all cases we will find that the average phase factor is nonzero even for large $\mu^{2} V$ provided that $2 \mu<m_{\pi}$. For $2 \mu>m_{\pi}$ the average phase is exponentially suppressed with $\mu^{2} V$.

Although QCD at nonzero baryon chemical potential has a sign problem, this is not the case for QCD with two colors, QCD with gauge fields in the adjoint representation and the phase quenched partition function. The chemical potential and mass dependence of these partition functions has been analyzed in great detail by means of chiral lagrangians or random matrix theory $9,10,14,21,22,23,24,25,26$ as well as on the lattice 27, 28, 29]. The success of these calculations suggests 
that equally impressive lattice QCD results can be obtained for the average phase factor. We hope that the results presented in this letter will encourage such calculations.

The approach introduced in this letter is directly applicable to QCD at nonzero $\theta$-angle. Fermion sign problems also appear in other interesting physical systems [30]. It would be worthwhile to analyze them along the lines proposed in this letter.

General arguments. In this section we will evaluate the $\mu$-dependence of the average phase factor in the mean field limit. Below we will confirm these results from the asymptotic limit of the exact expressions for the microscopic domain. In this domain, it is natural to work at fixed topology instead of fixed $\theta$-angle. The results presented in this section are for the thermodynamic limit and do not depend on the topological charge.

The vacuum energy density does not depend on the chemical potential in a phase that is not sensitive to the boundaries. This is the case in the normal phase where the chemical potential is below the mass of the lightest particle with the corresponding charge. For larger $\mu$ there is a net particle flux in the time direction of the Euclidean torus and the free energy depends on the chemical potential 31]. Although in the normal phase the free energy is $\mu$-independent, the excitations of the vacuum are not. For a chiral Lagrangian the masses of the Goldstone modes for $\mu<m_{\pi} / 2$ are given by [10]

$$
M(\mu)=m_{\pi}-b \mu,
$$

where $b$ is the charge of the particles corresponding to $\mu$. In the zero momentum sector, the thermodynamic limit of the partition function is therefore given by

$$
Z=J \prod_{k} \frac{1}{m_{\pi}-\mu b_{k}} e^{-V F},
$$

where the Jacobian, $J$, is from the measure of the Goldstone manifold and $F$ is the vacuum energy density, both evaluated at the saddle point. The prefactor gives a $1 / \mathrm{V}$ correction to the free energy density. The prefactor is important if we consider the expectation value of the phase factor of the quark determinant which is given by the ratio of two partition functions

$$
\left\langle e^{2 i \theta}\right\rangle_{N_{f}}=\frac{Z_{N_{f}+1 \mid 1^{*}}}{Z_{N_{f}}} .
$$

They are defined by $(\langle\cdots\rangle$ refers to quenched averaging $)$

$$
Z_{N_{f}+1 \mid 1^{*}}=\left\langle\frac{\operatorname{det}\left(D+\mu \gamma_{0}+m\right)}{\operatorname{det}\left(D^{\dagger}+\mu \gamma_{0}+m\right)} \operatorname{det}^{N_{f}}\left(D+\mu \gamma_{0}+m\right)\right\rangle,
$$

and

$$
Z_{N_{f}}=\left\langle\operatorname{det}^{N_{f}}\left(D+\mu \gamma_{0}+m\right)\right\rangle
$$

The partition function (6) contains [35] $N_{f}+1$ fermionic quarks and one conjugate bosonic quark. Assuming maximum spontaneous breaking of the axial flavor symmetry, this results in $N_{f}+1$ charged fermionic Goldstones composed of a fermionic quark and a conjugate bosonic anti-quark as well as an equal number of anti-particles with the opposite charge. In addition, for topological charge zero, we have the usual $\left(N_{f}+1\right)^{2}$ neutral bosonic Goldstones and one neutral Goldstone made out of two bosonic quarks. The partition function in the denominator contains $N_{f}^{2}$ neutral Goldstones. In the normal phase, the saddle point of the static part of the effective Lagrangian is $\mu$-independent and neither the Jacobian nor the free energy do depend on $\mu$. Using (4), the average phase factor for $\mu<m_{\pi} / 2$ is given by

$$
\left\langle e^{2 i \theta}\right\rangle_{N_{f}}=\frac{\left(m_{\pi}^{2}-4 \mu^{2}\right)^{N_{f}+1}}{m_{\pi}^{2 N_{f}+2}}=\left(1-\frac{4 \mu^{2}}{m_{\pi}^{2}}\right)^{N_{f}+1} .
$$

We emphasize that the free energies of $Z_{N_{f}+1 \mid 1^{*}}$ and $Z_{N_{f}}$ cancel. Hence for $\mu<m_{\pi} / 2$ the sign problem is not exponentially hard in the microscopic domain (2).

This is not the case for $\mu>m_{\pi} / 2$, where the free energy of $Z_{N_{f}+1 \mid 1^{*}}$ is $\mu$-dependent exactly as in other theories with charged Goldstone particles [10]. This leads to an exponential suppression of the average phase factor ( $F_{\pi}$ is the pion decay constant)

$$
\left\langle e^{2 i \theta}\right\rangle_{N_{f}} \sim e^{-2 V F_{\pi}^{2} \mu^{2}\left(1-m_{\pi}^{2} / 4 \mu^{2}\right)^{2}}
$$

for $\mu>m_{\pi} / 2$. In addition, the Goldstinos with mass $m_{\pi}-2 \mu$ become exactly massless for $\mu>m_{\pi} / 2$ [10] so that the leading contributions to the prefactor cancel. We conclude that the sign problem is not tractable for large $\mu^{2} F_{\pi}^{2} V$ and $\mu>m_{\pi} / 2$.

Microscopic result. As we have seen in Eq. (5), the expectation value of the phase factor is given by the ratio of two partition functions. We now calculate them in the microscopic limit where the scaling variables

$$
\hat{m}=m \Sigma V \quad \text { and } \quad \hat{\mu}=\mu F_{\pi} \sqrt{V}
$$

are kept fixed for $V \rightarrow \infty$. In this limit, the QCD partition function is equivalent to the large $N$ limit of a random matrix theory of $2 N \times 2 N$ matrices with the same global symmetries and transformation properties 12, 13]. This allows us to perform the calculations using recent developments in the method of orthogonal polynomials [17, 18, 19, 20]. Starting from a general expression in [19], it can be shown that the microscopic limit of the partition functions in (5) can be expressed in terms of modified Bessel functions and their Cauchy transforms. For zero topological charge we obtain (with $\delta_{\hat{m}}=\hat{m} d / d \hat{m}$ )

$$
\left\langle e^{2 i \theta}\right\rangle_{N_{f}} \sim
$$




$$
\frac{1}{Z_{N_{f}}} \frac{1}{\hat{m}^{N_{f}\left(N_{f}+1\right)}}\left|\begin{array}{lll}
X^{(0)}(\hat{m} ; \hat{\mu}) & \cdots & X^{\left(N_{f}+1\right)}(\hat{m} ; \hat{\mu}) \\
I_{0}(\hat{m}) & \cdots & \delta_{\hat{m}}^{N_{f}+1} I_{0}(\hat{m}) \\
\vdots & & \vdots \\
\delta_{\hat{m}}^{N_{f}} I_{0}(\hat{m}) & \cdots & \delta_{\hat{m}}^{2 N_{f}+1} I_{0}(\hat{m})
\end{array}\right|,
$$

where 11

$$
Z_{N_{f}} \sim \hat{m}^{-N_{f}\left(N_{f}-1\right)} \operatorname{det}\left[\delta_{\hat{m}}^{k+l} I_{0}(\hat{m})\right]_{k, l=0, \ldots, N_{f}-1} .
$$

The Cauchy transforms $X^{(k)}(\hat{m} ; \hat{\mu})$ are defined by

$$
X^{(k)}(\hat{m} ; \hat{\mu}) \equiv-\frac{e^{-2 \hat{\mu}^{2}}}{4 \pi \hat{\mu}^{2}} \int \mathrm{d}^{2} z \frac{w\left(z, z^{*}, \hat{\mu}\right) \delta_{z^{*}}^{k} I_{0}\left(z^{*}\right)}{z^{2}-\hat{m}^{2}}
$$

where $w\left(z, z^{*} ; \mu\right)$ is the weight function of the random matrix model in 17. The expressions for the Cauchy transform (13) can be rewritten as a one-dimensional integral following the approach of 21]. Next we give explicit results for the thermodynamic limit of (11) which is obtained from the saddle point approximation for $\hat{\mu} \rightarrow \infty$ and $\hat{m} \rightarrow \infty$ at fixed $\mu^{2} / m$.

In the quenched case $\left(N_{f}=0\right)$ a saddle-point approximation of (11) gives

$$
\left\langle e^{2 i \theta}\right\rangle_{N_{f}=0}=\left(1-\frac{4 \mu^{2}}{m_{\pi}^{2}}\right) e^{0}, \quad 2 \mu<m_{\pi} .
$$

This result agrees with the mean field arguments given above. For $\mu>m_{\pi} / 2$ the result is exponentially suppressed exactly as in (9) and with a prefactor that cancels to leading order in $1 / V$. Notice that the phase factor is only exponentially suppressed for $\mu>m_{\pi} / 2$.

For $N_{f}=1$ the thermodynamic limit of the exact microscopic result (11) is given by

$$
\left\langle e^{2 i \theta}\right\rangle_{N_{f}=1}=\left(1-\frac{4 \mu^{2}}{m_{\pi}^{2}}\right)^{2} e^{0}, \quad 2 \mu<m_{\pi},
$$

in agreement with the mean field arguments given above. For $\mu>m_{\pi} / 2$, at finite volume the result is given by (9) with a prefactor that cancels to leading order in $1 / V$.

By now it should be clear that the thermodynamic limit of the microscopic result (11) reproduces the general formula (8) for all values of $N_{f}$. As further illustration we plot in Fig. 1 the average phase factor for $N_{f}=2$. The dashed curve represents the result of Eq. (11) for $m V \Sigma=4$ and the full curve is its limit for $m V \Sigma \rightarrow \infty$ at fixed $\mu / m_{\pi}$. We observe a rapid convergence to the thermodynamic limit especially at small $\mu$.

Finally, we calculate the average phase factor for the phase quenched theory where the phase factor of the dynamical fermions is ignored. For two flavors it can be expressed as

$$
\left\langle e^{2 i \theta}\right\rangle_{1+1^{*}}=\frac{\left\langle\operatorname{det}^{2}\left(D+\mu \gamma_{0}+m\right)\right\rangle}{\left\langle\left|\operatorname{det}\left(D+\mu \gamma_{0}+m\right)\right|^{2}\right\rangle} .
$$

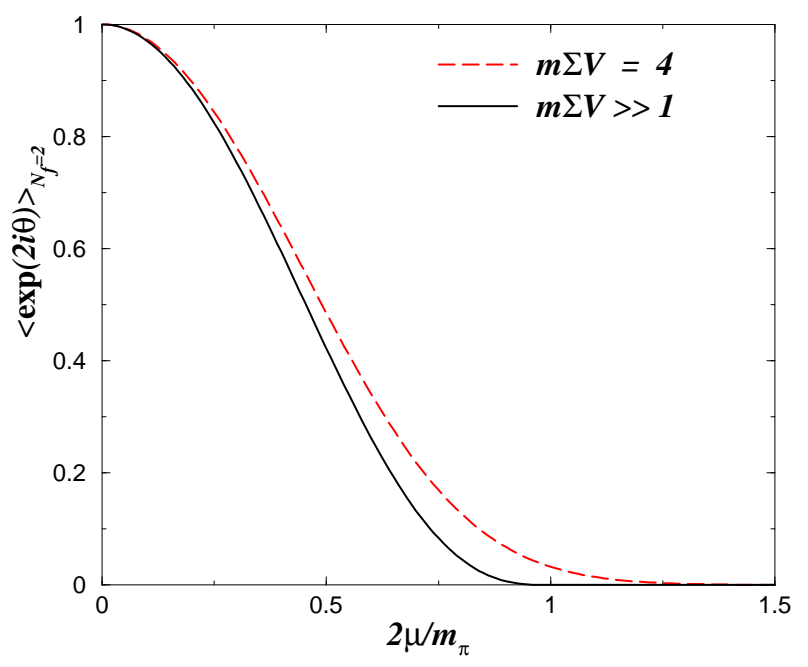

FIG. 1: The average phase factor for two flavor QCD in the $\epsilon$-regime as a function of the chemical potential for $m \Sigma V=4$ (dashed curve) and $m \Sigma V \rightarrow \infty$ (full curve).

The microscopic limit of both partition functions is wellknown [11, 23], resulting in the average phase factor

$$
\left\langle e^{2 i \theta}\right\rangle_{1+1^{*}}=\frac{I_{0}^{2}(\hat{m})-I_{1}^{2}(\hat{m})}{2 e^{2 \hat{\mu}^{2}} \int_{0}^{1} d t t e^{-2 \hat{\mu}^{2} t^{2}} I_{0}(\hat{m} t)^{2}} .
$$

In the thermodynamic limit obtained by making a saddle point approximation of the integrals in (17) we find the same result as in the quenched case (14). For $2 \mu>m_{\pi}$ the result is once more given by (9) but with a different prefactor than in the previous two cases.

Lattice Simulations. Several lattice simulations have studied the fluctuations of the phase of the fermion determinant. Both $\langle\cos \theta\rangle[32]$ and $\left\langle\theta^{2}\right\rangle[5,33$, were considered. In [5, 33] the variance of the phase of the fermion determinant was calculated using the Taylor expansion technique. To lowest order in an expansion in $\mu$ the average squared phase is given by

$$
\left\langle\theta^{2}\right\rangle-\langle\theta\rangle^{2}=\left.\mu^{2} \partial_{\mu_{1}} \partial_{\mu_{2}} \log Z_{1+1^{*}}\right|_{\mu_{1}=\mu_{2}=0},
$$

where

$$
Z_{1+1^{*}}=\left\langle\operatorname{det}\left(D+\mu_{1} \gamma_{0}+m\right) \operatorname{det}\left(D^{\dagger}+\mu_{2} \gamma_{0}+m\right)\right\rangle .
$$

In the microscopic limit, this partition function is given by the the denominator of Eq. (17) with $\mu \rightarrow\left(\mu_{1}-\mu_{2}\right) / 2$. In the thermodynamic limit we obtain $\left(\mu<m_{\pi} / 2\right)$

$$
\left\langle\theta^{2}\right\rangle-\langle\theta\rangle^{2}=2 \frac{\mu^{2}}{m_{\pi}^{2}}+\ldots .
$$

The work of Allton et al. [5, 33] indeed suggests that $\left\langle\theta^{2}\right\rangle \sim \mu^{2} / m_{\pi}^{2}$ but the prefactor appears to be several times larger than given by (201). There can be several reason for this discrepancy. First, the calculations of Allton et al. [5] were performed close to the critical temperature whereas our results are for zero temperature. 
In particular, susceptibilities are expected to be sensitive to the temperature. Second, our results have been derived for the $\epsilon$-domain of QCD whereas the pion mass in 5] does not satisfy the condition (2). Third, there could be significant ultra-violet contributions to the average squared phase. Although, it can be shown along the lines of 26] that in dimensional regularization the $\mu$-dependent terms do not introduce additional ultraviolet divergences, for a lattice regularization this is only the case after the necessary subtractions have been made. Ultra-violet contributions to the average phase factor are expected to behave as $\exp \left(-V w^{2} \Lambda^{2}\right)$ with $w$ the width of the strip of eigenvalues and $\Lambda$ an ultra-violet cut-off. Since $w \sim \mu^{2}$ (see [14]) ultra-violet contributions are suppressed in the microscopic limit.

Conclusions. We have shown that for sufficiently small $\mu$ the expectation value of the phase factor of the quark determinant can be obtained from chiral perturbation theory. Explicit expressions have been obtained in the microscopic domain where $\mu \sim 1 / \sqrt{V}$ and $m \sim 1 / V$. Our results show a phase transition of $\langle\exp (2 i \theta)\rangle$ at $\mu=m_{\pi} / 2$ with $\langle\exp (2 i \theta)\rangle=0$ beyond this point. In particular, this implies that there is no serious sign problem for $\mu \sim$ $1 / \sqrt{V}$ and a physical quark mass. However, the sign problem is severe in the chiral limit for any nonzero $\mu$ where it is essential for the discontinuity of the chiral condensate 34].

Our results have been derived for zero temperature. From the temperature dependence of the grand potential we expect that phase fluctuations initially increase with temperature. A deeper understanding of the sign problem could be obtained by extending the current lattice simulations to lower temperatures and quark masses. We believe that the confirmation of the analytical results for the phase fluctuations predicted in this letter will be an important step forward toward a first principles understanding of the QCD phase diagram at nonzero chemical potential.

Acknowledgments. We wish to thank G. Akemann, P.H. Damgaard, P. de Forcrand, M. Lüscher, D. Dietrich, J. Osborn and L. Ravagli for valuable discussions. This work was supported by U.S. DOE Grant No. DE-FG88ER40388 (JV) and the Carlsberg Foundation (KS).

[1] R. D. Pisarski and F. Wilczek, Phys. Rev. D 29, 338 (1984).

[2] U. M. Heller, plenary review PoS LAT2006.

[3] K. Rajagopal and F. Wilczek, hep-ph/0011333

[4] P. de Forcrand and O. Philipsen, Nucl. Phys. B 642, 290 (2002); Nucl. Phys. B 673, 170 (2003).

[5] C. R. Allton et al., Phys. Rev. D 66, 074507 (2002); Phys. Rev. D 68, 014507 (2003); Phys. Rev. D 71, 054508 (2005).

[6] Z. Fodor and S. D. Katz, JHEP 0203, 014 (2002); JHEP
0404, 050 (2004)

[7] M. D'Elia and M. P. Lombardo, Phys. Rev. D 67, 014505 (2003).

[8] R. V. Gavai and S. Gupta, Phys. Rev. D 68, 034506 (2003).

[9] J.B. Kogut, M.A. Stephanov, and D. Toublan, Phys. Lett. B 464, 183 (1999).

[10] J.B. Kogut et al., Nucl. Phys. B 582, 477 (2000).

[11] H. Leutwyler and A. Smilga, Phys. Rev. D 46, 5607 (1992).

[12] E. V. Shuryak and J. J. M. Verbaarschot, Nucl. Phys. A 560, 306 (1993).

[13] J. J. M. Verbaarschot, Phys. Rev. Lett. 72, 2531 (1994).

[14] D. Toublan and J. J. M. Verbaarschot, Int. J. Mod. Phys. B 15, 1404 (2001).

[15] Y.V. Fyodorov, B. Khoruzhenko and H.J. Sommers, Ann. Inst. Henri Poincaré: Phys. Theor. 68, 449 (1998).

[16] G. Akemann, Phys. Rev. Lett. 80, 072002 (2002); J. Phys. A: Math. Gen. 36, 3363 (2003).

[17] J. C. Osborn, Phys. Rev. Lett. 93, 222001 (2004).

[18] M.C. Bergère, hep-th/0311227 hep-th/0404126

[19] G. Akemann and A. Pottier, J. Phys. A 37, L453 (2004).

[20] G. Akemann, J. C. Osborn, K. Splittorff and J. J. M. Verbaarschot, Nucl. Phys. B 712, 287 (2005).

[21] K. Splittorff and J.J.M. Verbaarschot, hep-th/0605143

[22] M. Stephanov, Phys. Rev. Lett. 76, 4472 (1996).

[23] K. Splittorff and J. J. M. Verbaarschot, Nucl. Phys. B 683, 467 (2004).

[24] P. H. Damgaard, et al. Phys. Rev. D 72, 091501 (2005); Phys. Rev. D 73, 074023 (2006); hep-th/0604054

[25] D. T. Son and M. A. Stephanov, Phys. Rev. Lett. 86, 592 (2001).

[26] K. Splittorff, D. Toublan, and J.J.M. Verbaarschot, Nucl. Phys. B 620, 290 (2002); Nucl. Phys. B 639, 524 (2002).

[27] G. Akemann and T. Wettig, Phys. Rev. Lett. 92, 102002 (2004) [Erratum-ibid. 96, 029902 (2006)]; J. C. Osborn and T. Wettig, PoS LAT2005, 200 (2005); J. Bloch and T. Wettig, Phys. Rev. Lett. 97, 012003 (2006).

[28] S. Hands et al., Eur. Phys. J. C 17, 285 (2000) J. B. Kogut et al., Phys. Rev. D 64, 094505 (2001); J. B. Kogut, D. Toublan and D. K. Sinclair, Nucl. Phys. B 642, 181 (2002); R. Aloisio et al., Phys. Lett. B 493, 189 (2000); S. Chandrasekharan and F. J. Jiang, Phys. Rev. D 74, 014506 (2006); S. Muroya, A. Nakamura and C. Nonaka, Phys. Lett. B 551, 305 (2003).

[29] G. Akemann and E. Bittner, Phys. Rev. Lett. 96, 222002 (2006).

[30] S. Chandrasekharan and U. J. Wiese, Phys. Rev. Lett. 83, 3116 (1999).

[31] J. B. Kogut, et al., Nucl. Phys. B 225, 93 (1983).

[32] Y. Sasai, A. Nakamura and T. Takaishi, Nucl. Phys. Proc. Suppl. 129, 539 (2004).

[33] S. Ejiri, Phys. Rev. D 69, 094506 (2004); Phys. Rev. D 73, 054502 (2006).

[34] J. C. Osborn, K. Splittorff and J. J. M. Verbaarschot, Phys. Rev. Lett. 94, 202001 (2005).

[35] The denominator in (6) cannot be written as a convergent bosonic integral. In order to achieve this the denominator and the numerator have to be multiplied [21] by $\operatorname{det}(D+$ $\left.\mu \gamma_{0}+m\right)$. However, this does not affect the result of the counting argument presented in this section. 